\title{
Position Reconstruction in Monolithic Block Detectors
}

\author{
Matthias Streun, Holger Nöldgen, Günter Kemmerling, Stefan van Waasen
}

\begin{abstract}
In high resolution PET systems the detector generally uses a scintillator which consists of individual pixel elements. The scintillation light of such a pixel element will be identified and thus the interaction is localized by the pixel position. Consequently, the delivered position of such a detector can only take discrete values.

A different approach is the monolithic scintillator detector. A continuous scintillator block spans over an area of several photodetector pixels and the position is reconstructed from the recorded light distribution. Manufacturing of this detector is easier and the sensitivity is generally higher as no scintillating material is wasted for optical isolation between the pixels. But the challenge is to find a dedicated algorithm in order to identify the interaction position with sufficient resolution.

We will present measurements of a monolithic scintillator detector $\left(21 \times 18 \times 10 \mathrm{~mm}^{3} \quad\right.$ LYSO $)$ and compare different reconstruction methods. Already a Least Square Optimization algorithm based on a rather simple model delivers a resolution similar to an Artificial Neural Network approach but which requires pre-registered data for training. The comparison of the resolution to that of a pixelated detector of similar size and $2 \times 2 \times 10 \mathrm{~mm}^{3}$ pixels shows the superior performance of the continuous block.
\end{abstract}

\section{INTRODUCTION}

$\mathrm{T}$ HE detector of a PET scanner has the task to stop a gamma particle emitted from a positron annihilation event and deliver time and position information. The gamma energy of $511 \mathrm{keV}$ leads to a rather low cross section which requires a massive detector and makes the scintillation detector the detector of choice. A typical PET scintillator as LSO requires a depth of about $8 \mathrm{~mm}$ in order to stop just $50 \%$ of a $511 \mathrm{keV}$ gamma flow. It is a common method for high spatial resolution scanners to subdivide the scintillator into pixels. Each pixel can either be read out by one individual photodetector element (one-to-one coupling) [1] or by spreading its light over an area of several detectors where the specific scintillator pixel is identified by the light distribution (light sharing) [2]. As a matter of fact these pixels end up as thin rods or needles with a small footprint $\left(\leq 2 \times 2 \mathrm{~mm}^{2}\right)$ but the full depth $(\geq 8 \mathrm{~mm})$ in order to maintain the sensitivity. Unfortunately, the higher the required resolution and the thinner the pixels, the more of the detector volume consists of optical isolation instead of scintillating material and a reduced sensitivity is the result. Furthermore, the manufacturing and assembling becomes more difficult and also cost intensive.

Manuscript received November 16, 2012

All authors are with Zentralinstitut für Elektronik, Forschungszentrum Jülich GmbH, Jülich, Germany (telephone: +49-2461-61-3182, e-mail: m.streun@fz-juelich.de)
A way out could be the monolithic block detector which has been subject to several papers during the last years [3-5]. One continuous scintillator block is placed on a pixelated photo detector and the position is reconstructed from the light distribution on the pixels by a dedicated algorithm which even can provide the depth of interaction information [6].

In this work two different positioning algorithms where evaluated. The first is an Artificial Neural Network approach (ANN) which requires a training process with prerecorded data but has the ability to adapt to imperfections of the individual detector as inhomogeneous optical coupling or scintillator light yield or non-uniform photodetector gain. The second algorithm is a Least Square Optimization (LS) based on the model that each scintillation event is localized in a single interaction point and that the signal of each photodetector element is proportional to the solid angle covered by its area [7].

\section{SETUP}

The two detector heads under investigation show similar sizes: The continuous head is an LYSO block with the dimensions $21.4 \times 18.5 \times 10 \mathrm{~mm}^{3}$. The pixelated head consists of an $8 \times 8$ matrix of $2 \times 2 \times 10 \mathrm{~mm}^{3}$ LYSO crystals embedded in a $\mathrm{BaSO}_{4}$ mounting. The gap between the crystals is $0.3 \mathrm{~mm}$ which leads to outer boundaries for the sensitive volume of $18.1 \times 18.1 \times 10 \mathrm{~mm}^{3}$. Figure 1 shows a photo of both heads. Width and height of the heads are almost identical, but we chose the continuous head to be longer. In this way we can also investigate the performance of a continuous block protruding over the edges of the photo detector area. This will be of special interest for a PET scanner where a close arrangement of the detector heads is required.

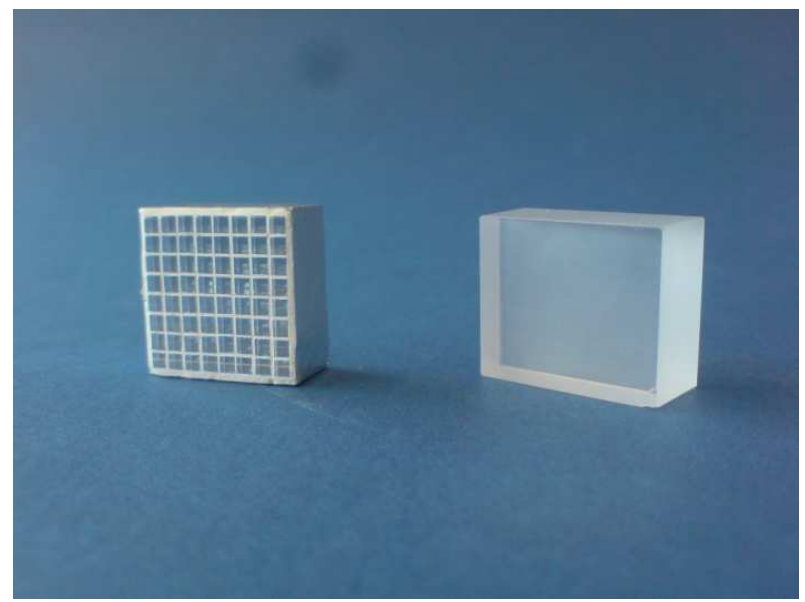

Fig. 1: Pixelated scintillator matrix and monolithic LYSO block. 


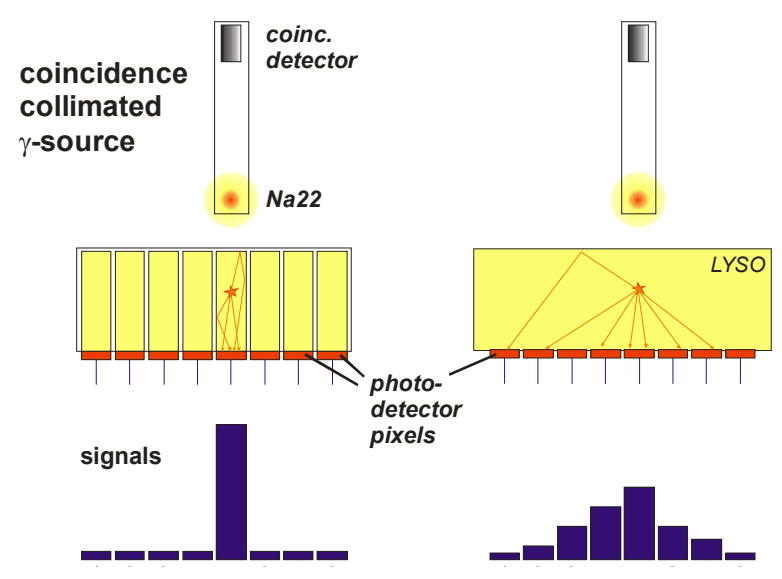

Fig. 2. Schematic drawing of a section through the setup for the pixelated detector (left) and the continuous crystal (right). Typical signal distributions are given below.

The measurements for both heads are performed with the same setup. The detector head is coupled by an optical gel to an $8 \times 8$ channel photomultiplier (PMT) (Hamamatsu 7600M64). Pixel size and pitch of the PMT is identical to that of the pixelated head which allows for a one-to-one coupling of the pixels.

PMT and head can be moved by an xy-positioning table in the horizontal plane. A Na22 source of $0.25 \mathrm{~mm}$ active diameter is fixed above the head and aligned with a coincidence detector (see fig. 2). The readout electronics detect coincident events between this detector and the PMT for electronic collimation. A diode matrix sums the 64 PMT channels to eight row and eight column signals and a chargeto-time converter [8] translates them into pulse lengths. After conversion by a time-to-digital converter (TDC) the 16 values are transmitted to a pc for recording in case that a coincidence was detected.

The electronically collimated beam has a FWHM of approximately $1.0 \mathrm{~mm}$ at the surface of the scintillator head.

\section{MEASUREMENTS}

The surfaces of the detector heads were scanned in an equidistant grid. For the continuous head we chose a grid spacing of $1 \mathrm{~mm}$ running from $-10 \mathrm{~mm}$ to $+10 \mathrm{~mm}$ in $\mathrm{x}$-dimension and $-9 \mathrm{~mm}$ to $+9 \mathrm{~mm}$ in $\mathrm{y}$-direction. We recorded more than 1200 events in each position. Each event consists of the eight row and eight column values representing the light distribution on the PMT. The pixelated head was scanned with a finer grid of $0.5 \mathrm{~mm}$ for a better representation of the individual scintillator pixels. The grid range reached from $-9 \mathrm{~mm}$ to $+9 \mathrm{~mm}$ in both dimensions.

\section{METHODS}

\section{A. Energy Selection}

First, all recorded data have to pass an energy window. To this end an energy spectrum is generated from the pixel sums of each event. Only those events are selected that belong to the range of the $511 \mathrm{keV}$ photo peak that corresponds to a window of approx. $300-700 \mathrm{keV}$.

In case of the pixelated head we created the spectrum and applied the energy window separately for each individual crystal. This was necessary because the individual pixels vary in light output, optical coupling and gain of the PMT channel.

\section{B. Position Determination}

\section{1) Pixelated Detector}

The majority of the gamma particles detected by the pixelated head generate a signal only in one single scintillator pixel. Thus, the center position of this pixel is defined as the detected position of this event. Events that give response in multiple pixels are discarded. In this case the crystal identification can be ambiguous due to the summed row and column signals. In general those events are induced by intercrystal scattering. Their occurrence is rather low $(<5 \%)$ and the contribution to the final result would be negligible.

Differently to that the continuous head produces rather broad light distributions and in general several pixels of the photodetector will respond when a gamma particle hits the scintillator crystal. In order to decode the distribution and get the original interaction position we made use of two different algorithms.

\section{2) Artificial Neural Networks (ANN)}

According to the read out architecture which combines the pixel signals to rows and columns we employ two separate independent networks, one for each dimension. The networks show a plain topology and are realized as feed forward networks with 8 inputs, one output and two hidden layers with 5 neurons each. For their implementation we used the software tool Mathematica (Wolfram Research Inc.).

In a first step the recorded data of each event were normalized by dividing each row and each column value by the sum of all 16 row and column values. Next the data were divided in a training data set and an evaluation data set. For the training data we took a fixed number of events $(=400)$ from each source position. The evaluation data set consists of the remaining events. In order to avoid errors that may be caused by drifting components or parameters during the acquisition we didn't simply take the first or last recorded subsequent events but picked the events randomly from the data of each source position.

The training of the ANNs is based on the LevenbergMarquardt algorithm. All events of the training data set together with their true source positions were used as input. After being trained the ANNs deliver the coordinates of a position when fed with the light distribution pattern of an event. Ideally, this position should reproduce the source position in which the light distribution has been recorded.

\section{3) Least Square Optimization (LS)}

In contrary to the ANNs this algorithm is based on a physical model and obtains the source position by an optimization process that minimizes a cost function. It had been introduced by Zhi et al. [7]. The model assumes a single interaction point $(x, y, z)$ within the scintillator as an isotropic light source. Each 
photodetector pixel obtains a part of this light which depends on the solid angle given by the pixels sensitive area:

$$
N p h_{n m}=C_{0}+A_{0} \times \Omega_{x, y, z, n m}
$$

where $N p h_{n m}$ is the number of photons on the pixel in row $n$ column $m$. $A_{0}$ is the total amount of light emitted and $\Omega$ the solid angle from the interaction point $(x, y, z)$ covered by pixel $\mathrm{nm} . C_{0}$ represents diffuse light that gets reflected on the crystal surfaces. Regular reflections from polished surfaces would have to be taken into account by additional mirror source terms [7], but this is not the case for the scintillator in our setup which has rough side faces.

The solid angle can be approximated to

$$
\Omega_{x, y, z, n m}=\frac{a \times z}{\left(\left(x-x_{n}\right)^{2}+\left(y-y_{m}\right)^{2}+z^{2}\right)^{3 / 2}}
$$

where $a$ is the pixel area and $\left(x_{n}, y_{m}\right)$ are the center coordinates of pixel $\mathrm{nm}$. The approximation is valid only for large distances between interaction point and pixel, but simulation studies showed that the deviation from an exact calculation is negligible [7].

Finally, the unknown position $(x, y, z)$ together with the arguments $C_{0}$ and $A_{0}$ are obtained by minimizing the cost function:

$$
\begin{aligned}
\left(x, y, z, C_{0}, A_{0}\right) & \\
=\underset{\left(x, y, z, C_{0}, A_{0}\right)}{\arg \min }( & \sum_{n=1}^{8}\left(\operatorname{row}_{n}-\sum_{m=1}^{8} N p h_{n m}\right)^{2} \\
& \left.+\sum_{m=1}^{8}\left(\operatorname{col}_{m}-\sum_{n=1}^{8} N p h_{n m}\right)^{2}\right) .
\end{aligned}
$$

The experimental data provide the light distribution on the pixels as eight row values row $_{n}$ and eight column values $\operatorname{col}_{m}$ and consequently the pixel intensities $N p h_{n m}$ have to be summed in order to generate the corresponding row and column intensities. The experimental data $\left(\right.$ row $_{n}$, col $\left._{m}\right)$ are normalized in the same manner as done for the ANNs. We used the Matlab optimization toolbox to find the local minimum when choosing the initial values for $(x, y)$ at the row and column with the largest signal. The initial value for $z$ was set to $6 \mathrm{~mm}$, for $A_{0}$ to 0.8 and for $C_{0}$ to $\left(\min \operatorname{row}_{n}+\min \operatorname{col}_{m}\right) / 16$ which is an estimate for the weakest pixel signal.

\section{Evaluation}

Finally, for the $N$ event data sets $\{\text { row }, c o l\}_{i}$ in source position $S=\left(x_{S}, y_{S}\right)$ the positioning algorithm generates the $N$ suggested positions $p_{i}=\left(x_{p}, y_{p}\right)_{i}$. The better the $p_{i}$ represent the source position $S$, the higher is the quality of the positioning. A common method to describe the positioning of a detector is to present the statistical error of the distribution as $\operatorname{FWHM}\left\{p_{i}\right\}$ and FWTM $\left\{p_{i}\right\}$ and the systematic error as the difference between the peak position of $\left\{p_{i}\right\}$ and the position of the source $S$. But this assumes a distribution with a single peak which is not always the case. Events in side peaks are ignored. Furthermore it requires a 2-dimensional Gaussian fit which can be ambiguous because the result strongly depends on the starting values and the range of data included.

For a fair comparison of the different detectors and algorithms within this work we chose the Root Mean Square Deviation (RMSD) as a measure for the positioning quality. It combines statistical and systematic error and takes all events into account. For each source position $S$ the RMSD is calculated from

$$
R M S D_{S}=\sqrt{\sum_{i}^{N}\left(S-p_{i}\right)^{2} / N} .
$$

In general the RMSD produces much larger values than the FWHM.

\section{RESULTS}

The RMSD values of the $21 \times 19$ source positions for the continuous detector are displayed as color coded maps in figure 3 for the LS and the ANN algorithm. The dimensions of the maps represent the surface of the detector. Both algorithms show a very similar distribution of the RMSD that is within a range of $2.5 \mathrm{~mm}$ to $3.5 \mathrm{~mm}$ in the center area and starts to increase approximately $2 \mathrm{~mm}$ from the edges. Averaged over the total detector the RMSD is $3.8 \mathrm{~mm}$ for both LS and ANN positioning.

Fig. 4 shows the corresponding map for the pixelated detector and had been created from RMSD values in $37 \times 37$ source positions. The individual scintillator pixels can be identified clearly. The pixelated detector delivers discrete positions only which are given by the center positions of the pixels. This causes an intrinsic positioning deviation for any event not occurring at the center of a pixel. As a result the overall performance is worse compared to the continuous detector and shows an average RMSD of $4.5 \mathrm{~mm}$.

\section{CONCLUSION}

Two methods of event positioning in monolithic block detectors were tested. In contrary to the ANN positioning the LS algorithm is based on a physical model and does not have the ability to adapt to the individual properties of the detector. Nevertheless the precision of both methods show very similar results. This is very promising as ANNs are not very practical when implemented in large systems due to the necessary training procedure.

In any case the monolithic block provided far better results than the pixelated scintillator with $2 \times 2 \mathrm{~mm}^{2}$ pixels, when both had a thickness of $10 \mathrm{~mm}$. 

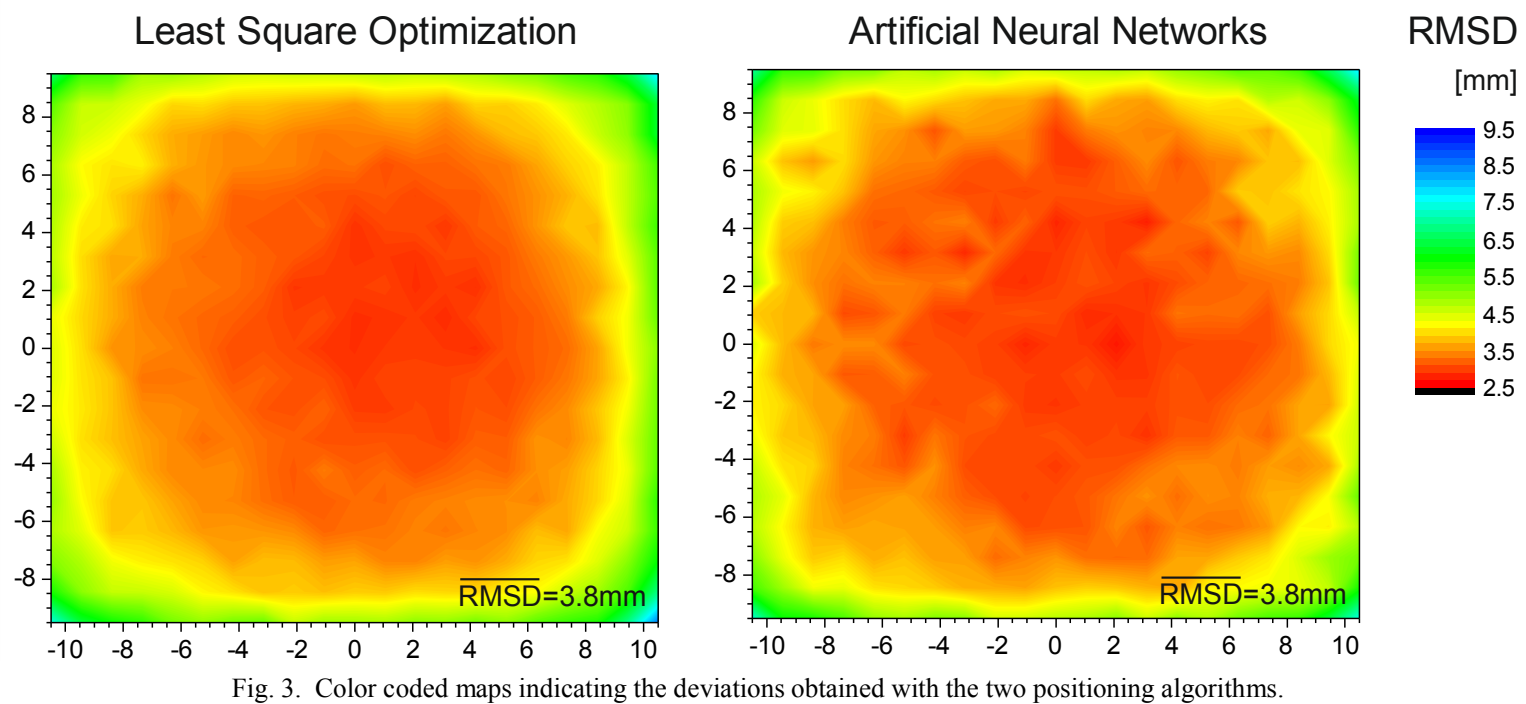

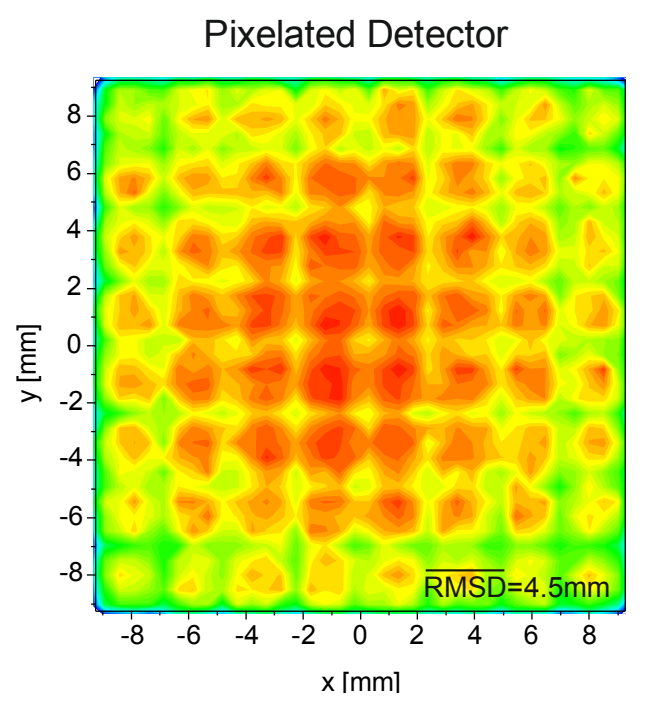

Fig. 4. Map of the RMSD for the pixelated detector. The color coding is equal to figure 3 .

\section{REFERENCES}

[1] K. Ziemons et al.," The ClearPET ${ }^{\mathrm{TM}}$ project: development of a 2nd generation high-performance small animal PET scanner", Nucl. Inst. Meth. A vol. 537, p. 307, 2005

[2] R. A. Mintzer and S. B. Siegel, "Design and performance of a new pixelated-LSO/PSPMT gamma-ray detector for high resolution PET imaging”, 2007 IEEE NSS Conf. Rec. p. 3418, 2007

[3] S. Delorme, R. Frei, C. Joseph, J.-F. Laude, and C. Morel, "Use of a neural network to exploit light division in a triangular scintillating crystal", Nucl. Instr. Meth. A, vol. 373, p. 111, 1996

[4] P. Bruyndonckx, S. Leonard, S. Tavernier, C. Lemaitre, O. Devroede, Yibao Wu, M. Krieguer, "Neural network-based position estimators for PET detectors using monolithic LSO blocks", IEEE Trans. Nucl. Sci. vol. 51 (5), p. 2520,2004

[5] G. Llosa et al., "Characterization of a PET detector head based on continuous LYSO crystals and monolithic, 64-pixel silicon photomultiplier matrices" Phys. Med. Biol., vol. 55, p. 7299, 2010

[6] M.C. Maas, D.R. Schaart, D.J. van der Laan, P. Bruyndonckx, C. Lemaître, F.J. Beekman, and C.W.E. van Eijk, "Monolithic scintillator PET detectors with intrinsic depth-of-interaction correction”, Phys. Med. Biol. vol. 54, p. 1893, 2009

[7] C. Parl, H.Larue, M.Streun, K.Ziemons and S. van Waasen, "Fast Charge to Pulse Width Converter for Monolith PET Detector", Trans. Nucl. Sci. vol. 59 (5), p. 1809, 2012

[8] Zhi Li, M. Wedrowski, P. Bruyndonckx and G. Vandersteen, "Nonlinear least-squares modeling of 3D interaction position in a monolithic scintillator block", Phys. Med. Biol., vol. 55, p. 6515, 2010 